\title{
Shortcuts to Adiabaticity in Driven Open Quantum Systems: Balanced Gain and Loss and Non-Markovian Evolution
}

\author{
S. Alipour*1, A. Chenu*2,3, A. T. Rezakhani ${ }^{4}$, and A. del Campo ${ }^{2,3,5}$ \\ ${ }^{1}$ QTF Center of Excellence, Department of Applied Physics, Aalto University, P. O. Box 11000, Fl-00076 Aalto, Espoo, Finland \\ ${ }^{2}$ Donostia International Physics Center, E-20018 San Sebastián, Spain \\ ${ }^{3}$ IKERBASQUE, Basque Foundation for Science, E-48013 Bilbao, Spain \\ ${ }^{4}$ Department of Physics, Sharif University of Technology, Tehran 14588, Iran \\ ${ }^{5}$ Department of Physics, University of Massachusetts, Boston, MA 02125, USA
}

A universal scheme is introduced to speed up the dynamics of a driven open quantum system along a prescribed trajectory of interest. This framework generalizes counterdiabatic driving to open quantum processes. Shortcuts to adiabaticity designed in this fashion can be implemented in two alternative physical scenarios: one characterized by the presence of balanced gain and loss, the other involves non-Markovian dynamics with time-dependent Lindblad operators. As an illustration, we engineer superadiabatic cooling, heating, and isothermal strokes for a two-level system, and provide a protocol for the fast thermalization of a quantum oscillator.

Shortcuts to adiabaticity (STA) allow controlling the evolution of a quantum system without the requirement of slow driving [1-3]. The controlled speedup of quantum processes is broadly recognized as a necessity for the advance of quantum technologies, and STA have found a variety of applications, including phase-space preserving cooling [4], population transfer [5, 6], and friction suppression in finite-time thermodynamics [7-9], to name some relevant examples. To date, STA have been demonstrated in the laboratory using ultracold gases [1016], nitrogen-vacancy centers [17, 18], trapped ions [19], superconducting qubits [20, 21], and other systems [1].

Despite this remarkable progress, the use of STA has been predominantly restricted to tailor the dynamics of isolated driven systems. However, any physical system is embedded in a surrounding environment with which it can interact and exchange en-

\footnotetext{
${ }^{*}$ These authors contributed equally to the work.
}

ergy, particles, etc. In such a setting, the dynamics of the system is no longer-described by a Hamiltonian and is associated with a master equation [22]. A notable exception concerns the dynamics of an isolated system conditional to a given subspace of interest. The dynamics can then be described in terms of a non-Hermitian Hamiltonian, that generates loss and gain when the system leaves the subspace of interest and returns to it, respectively [23]. Scenarios characterized by a balance of gain and loss arise naturally, e.g., in the presence of a non-Hermitian potential that breaks time-reversal symmetry but preserves paritytime-reversal symmetry, i.e., in $\mathcal{P} \mathcal{T}$-symmetric quantum mechanics [24-29].

Recent efforts on developing STA in open quantum systems have predominantly focused on mitigating decoherence $[1,3]$. Perturbative methods have been put forward to inhibit unwanted transitions in two- and three-level systems [30, 31], while the use of decoherence-free subspaces in open quantum systems allow one to mitigate decoherence [32-34],

However, the use of STA to speed up open quantum processes is expected to make possible a wide range of applications such as design of novel cooling techniques, information erasure [35], or the engineering of superadiabatic quantum machines [36]. In this context, the engineering of STA in systems described by non-Hermitian Hamiltonians has been advanced in Refs. [37-40] while the control by STA of arbitrary nonunitary dynamics requires further progress. A pioneering effort to this end introduced fast control protocols for Markovian processes [41]. This guarantees an independent evolution for the different Jordan blocks forming the Lindblad operator, thus fulfilling the notion of adiabaticity for open system introduced in Ref. [42]. More recently, the fast thermalization of a harmonic oscillator has been proposed via the reverse engineering of a non-adiabatic Markovian 
master equation [43] and engineered dephasing [44]. A related study has shown the possibility of speeding up the thermalization of a system oscillator locally coupled to a harmonic bath [45]. Engineering of the system-bath coupling has also been proposed to speed-up isothermal processes [46]. The fast driving between equilibrium and squeezed states has also been presented [47].

In this paper, we introduce a universal scheme to engineer STA in arbitrary open quantum systems. Our work provides a generalization of the counterdiabatic driving technique $[5,6,48]$ to open quantum processes. To this end, we consider the evolution of a quantum system described by a mixed state along a prescribed trajectory of interest. We then find the equation of motion that generates the desired dynamics. The latter can be recast in terms of the nonlinear evolution of a system in the presence of balanced gain and loss. Alternatively, the dynamics can be associated with a non-Markovian master equation with time-dependent Lindblad operators whose explicit form is determined by the prescribed trajectory. We demonstrated this framework by discussing the controlled open quantum dynamics of a two-level system and a driven quantum oscillator.

\section{STA by counterdiabatic driving}

Consider a quantum evolution of interest described by the mixed state

$$
\varrho(t)=\sum_{n=1}^{r} \lambda_{n}(t)\left|n_{t}\right\rangle\left\langle n_{t}\right|,
$$

of finite rank $r=\operatorname{rank}(\varrho)$. We pose the problem of enforcing the evolution of the system through this trajectory.

Under unitary dynamics, eigenvalues of the density matrix remain constant, $\lambda_{n}(t)=\lambda_{n}(0)$-denoted briefly as $\lambda_{n}$. The equation of motion for the density matrix in this case reads

$$
\partial_{t} \varrho(t)=\sum_{n} \lambda_{n}\left(\left|\partial_{t} n_{t}\right\rangle\left\langle n_{t}|+| n_{t}\right\rangle\left\langle\partial_{t} n_{t}\right|\right),
$$

and can be recast as a Liouville-von Neumann equation, $\partial_{t} \varrho(t)=-i\left[H_{1}(t), \varrho(t)\right]$ (with $\hbar=1$ ), whenever the dynamics is generated by the Hamiltonian

$$
H_{1}(t)=i \sum_{n}\left(\left|\partial_{t} n_{t}\right\rangle\left\langle n_{t}\left|-\left\langle n_{t} \mid \partial_{t} n_{t}\right\rangle\right| n_{t}\right\rangle\left\langle n_{t}\right|\right) .
$$

This Hamiltonian generates parallel transport along each of the eigenstates $\left|n_{t}\right\rangle$ and is often used in proofs of the adiabatic theorem $[49,50]$.
In the context of control theory, the derivation of $H_{1}(t)$ can be systematically achieved by the so-called counterdiabatic (CD) driving technique, also known as transitionless quantum driving [5, 6, 48]. Specifically, CD assumes that $\left|n_{t}\right\rangle$ are the eigenstates of a reference system $H_{0}(t)$ that can be controlled by the auxiliary field $H_{1}(t)$ so that the full dynamics is actually generated by $H_{0}(t)+H_{1}(t)$. Yet, in the most general setting, the instantaneous eigenstates used in the specification of the trajectory (1) need not be the eigenstates of the physical Hamiltonian of the system. To identify a reference Hamiltonian in this case, we choose $\varrho(t)$ to evolve as a thermal state,

$$
\varrho(t)=e^{-\beta H_{0}(t)} / Z_{0}(t),
$$

where $Z_{0}(t)=\operatorname{Tr}\left[e^{-\beta H_{0}(t)}\right]$ denotes the partition function, and $\beta$ is the inverse temperature (assuming $k_{\mathrm{B}}=1$ ). With this definition, the spectral decomposition of the reference Hamiltonian reads

$$
H_{0}(t)=\sum_{n} E_{n}\left|n_{t}\right\rangle\left\langle n_{t}\right|
$$

where the eigenvalues $E_{n}=-\beta^{-1} \log \left(Z_{0} \lambda_{n}\right)$ are time-independent, and so is the partition function. By construction $\left[H_{0}(t), \varrho(t)\right]=0$, and the state $\varrho(t)$ is a solution of

$$
\partial_{t} \varrho(t)=-i\left[H_{\mathrm{CD}}(t), \varrho(t)\right],
$$

where $H_{\mathrm{CD}}(t)=H_{0}(t)+H_{1}(t)$.

\section{CD driving of open quantum sys- tems}

In what follows we shall focus on the case where the eigenvalues of the density matrix are time-dependent. The von Neumann entropy of the state is then a function of time, and the dynamics is generally open and nonunitary. Indeed, for an arbitrary change of the eigenvalues $\left\{\lambda_{n}\right\}$ the dynamics is generally nontrace-preserving.

For a given time-dependence of $\lambda_{n}(t)$, the equation of motion for the trajectory $\varrho(t)$ can be analogously derived as

$$
\partial_{t} \varrho(t)=-i\left[H_{\mathrm{CD}}(t), \varrho(t)\right]+\sum_{n} \partial_{t} \lambda_{n}(t)\left|n_{t}\right\rangle\left\langle n_{t}\right| .
$$

The dynamics is trace-preserving whenever $\sum_{n} \lambda_{n}(t)=1$, i.e., $\sum_{n} \partial_{t} \lambda_{n}(t)=0$. The equation of motion (7) admits several physical interpretations that we discuss below. 


\subsection{Mixed evolution under balanced gain and} loss

The additional term in Eq. (7) can be associated with the anti-Hermitian operator

$$
-i \Gamma(t)=\frac{i}{2} \sum_{n} \frac{\partial_{t} \lambda_{n}(t)}{\lambda_{n}(t)}\left|n_{t}\right\rangle\left\langle n_{t}\right| .
$$

The equation of motion for $\varrho(t)$ is then generated by the full non-Hermitian Hamiltonian $H(t)=$ $H_{\mathrm{CD}}(t)-i \Gamma(t)$, i.e.,

$$
\begin{aligned}
\partial_{t} \varrho(t) & =-i\left(H(t) \varrho(t)-\varrho(t) H^{\dagger}(t)\right) \\
& =-i\left[H_{\mathrm{CD}}(t), \varrho(t)\right]-\{\Gamma(t), \varrho(t)\} .
\end{aligned}
$$

For arbitrary $\left\{\lambda_{n}\right\}$, this evolution is not necessarily norm-preserving and the norm varies at a rate

$$
\partial_{t} \operatorname{Tr}[\varrho(t)]=-2 \operatorname{Tr}[\Gamma(t) \varrho(t)]=\sum_{n} \partial_{t} \lambda_{n}(t) .
$$

A norm-preserving evolution through the trajectory $\varrho(t)$ is governed by the modified equation of motion

$$
\begin{aligned}
\partial_{t} \varrho & =-i\left(H \varrho-\varrho H^{\dagger}\right)-\partial_{t} \operatorname{Tr}[\varrho] \varrho \\
& =-i\left[H_{\mathrm{CD}}, \varrho\right]+(2\langle\Gamma\rangle \varrho-\{\Gamma, \varrho\}),
\end{aligned}
$$

where $\langle\Gamma\rangle=\operatorname{Tr}[\Gamma \varrho]$ and the time-dependence of all terms has been dropped for brevity. Note that the resulting equation is nonlinear in the quantum state $\varrho$. This dynamics thus takes the form of a mixed-state evolution in the presence of balanced gain and loss [51] with a time-dependent generator [52]. Balanced gain and loss arises naturally in the study of $\mathcal{P} \mathcal{T}$ symmetric quantum systems [24], that can be used to describe a variety of experiments [25-29].

\subsection{Lindblad-like form}

Considering the prescribed trajectory (1) and its derivative (7), one can recast the incoherent part

$$
\mathcal{D}_{\mathrm{CD}}(\varrho)=\sum_{n} \partial_{t} \lambda_{n}(t)\left|n_{t}\right\rangle\left\langle n_{t}\right|
$$

as an auxiliary CD dissipator in a Lindblad-like form for a trace-preserving trajectory. Assuming a tracepreserving evolution, $\sum_{n} \partial_{t} \lambda_{n}(t)=0$, we find the time-dependent Lindblad operators and rates as (see the appendixes)

$$
\begin{aligned}
L_{m n}(t) & =\left|m_{t}\right\rangle\left\langle n_{t}\right|, \\
\gamma_{m n}(t) & =\frac{\partial_{t} \lambda_{m}(t)}{r \lambda_{n}(t)},
\end{aligned}
$$

that are determined by (the spectral resolution of) $\varrho(t)$ - and thus state-dependent. The resulting master equation

$$
\begin{aligned}
\partial_{t} \varrho= & -i\left[H_{\mathrm{CD}}, \varrho\right] \\
& +\sum_{m n} \gamma_{m n}\left(L_{m n} \varrho L_{m n}^{\dagger}-\frac{1}{2}\left\{L_{m n}^{\dagger} L_{m n}, \varrho\right\}\right)
\end{aligned}
$$

is generally non-Markovian, because of possibly negative rates. We remark that the existence of a Lindblad-like master equation for an arbitrary dynamics has recently been proven in Ref. [53]. However, in the representation (7) like a Lindblad-like master equation, the anticommutator term in Eq. (14) identically vanishes and the dissipator reduces exclusively to jumps in the instantaneous eigenbasis.

The equivalence of Eqs. (11) and (14) shows that the nonlinear evolution of a mixed state under balanced gain and loss can be represented by a nonlinear and generally non-Markovian master equation with time-dependent Lindblad operators, determined by choice of the trajectory (1).

We note that the time-evolution operator generated by the CD Hamiltonian takes the form [48]

$$
U_{\mathrm{CD}}(t, 0)=\sum_{n} e^{i \phi_{n}(t)}\left|n_{t}\right\rangle\left\langle n_{0}\right|,
$$

where the time-dependent phase $\phi_{n}(t)$ is the sum of the dynamical and geometric contributions. In the co-moving frame associated to $U_{\mathrm{CD}}(t, 0)$, the master equation for $\tilde{\varrho}(t)=U_{\mathrm{CD}}^{\dagger}(t, 0) \varrho(t) U_{\mathrm{CD}}(t, 0)$ takes the simple form

$$
\partial_{t} \tilde{\varrho}=\sum_{m n} \gamma_{m n}\left(\tilde{L}_{m n} \tilde{\varrho} \tilde{L}_{m n}^{\dagger}-\frac{1}{2}\left\{\tilde{L}_{m n}^{\dagger} \tilde{L}_{m n}, \tilde{\varrho}\right\}\right),
$$

with $\tilde{L}_{m n}=\left|m_{0}\right\rangle\left\langle n_{0}\right|$. As a result, the timedependent Lindblad operators $\left\{L_{m n}\right\}$ map to the time-independent ones $\left\{\tilde{L}_{m n}\right\}$, while keeping the same rates $\gamma_{m n}(t)$. This feature is specific to the superadiabatic driving of open quantum systems and differs from the general case that leads to more complex time-dependent Lindblad operators [22].

\section{Quantum speed limit for STA in open quantum processes}

Time-energy uncertainty relations identify characteristic time scales in a physical process. Speed limits sharpen this identification by providing a minimum time for a physical processes to occur in terms of the generator of the evolution. We next show how speed limits relate the operation time of a protocol to 
the amplitude of the required unitary and nonunitary $\mathrm{CD}$ terms. The geometric formulation of the quantum speed limit [54] states that

$$
\tau \geqslant D(\varrho(0), \varrho(\tau)) /\left\langle\sqrt{g_{t t}}\right\rangle,
$$

where $g_{t t}$ is the metric for a given distance $D$, and the time average $\left\langle\sqrt{g_{t t}}\right\rangle_{\tau}=(1 / \tau) \int_{0}^{\tau} d t \sqrt{g_{t t}}$ upper bounds the speed of evolution.

The quantum Fisher information $\mathcal{F}$ is the metric (with a $1 / 4$ prefactor) associated with the Bures distance between quantum states

$$
D_{B}\left(\varrho_{1}, \varrho_{2}\right)=\left[2\left(1-F\left(\varrho_{1}, \varrho_{2}\right)\right]^{1 / 2},\right.
$$

that is defined in terms of the fidelity $F\left(\varrho_{1}, \varrho_{2}\right)=$ $\operatorname{Tr} \sqrt{\sqrt{\varrho_{1}} \varrho_{2} \sqrt{\varrho_{1}}}$ between $\varrho_{1}$ and $\varrho_{2}$ [55]. The speed limit (17) implies that the driving time of the process is constrained by the ratio of the distance between quantum states $D(\varrho(0), \varrho(\tau))$ and the velocity at which is traversed $\left\langle\sqrt{g_{t t}}\right\rangle$.

From Eq. (9), we can identify $-2 i H$ as a nonHermitian symmetric logarithmic derivative, satisfying $2 \partial_{t} \varrho=\mathbb{L} \varrho+\varrho \mathbb{L}^{\dagger}$ [56], based on which an upper bound on the quantum Fisher information is obtained as $\mathcal{F}=\operatorname{Tr}\left[\varrho \mathbb{L}^{2}\right] \leqslant 4 \operatorname{Tr}\left[H \varrho H^{\dagger}\right]$. As a result, the quantum speed limit reads

$$
\tau \geqslant \frac{D_{B}(\varrho(0), \varrho(\tau))}{4\left\langle\operatorname{Tr}\left[\left(H_{\mathrm{CD}}-i \Gamma\right) \varrho(t)\left(H_{\mathrm{CD}}+i \Gamma^{\dagger}\right)\right]^{1 / 2}\right\rangle_{\tau}} .
$$

The minimum time to implement a STA driving the system from $\varrho(0)$ to $\varrho(\tau)$ is thus not only governed by the Hermitian system Hamiltonian $H_{\mathrm{CD}}$, but as well by the term $\Gamma$ governing gain and loss.

Alternatively, using the trace distance rather than the Bures distance, the relevant metric is $g_{t t}=$ $\left\|\partial_{t} \varrho\right\|_{1}^{2} \equiv\left(\operatorname{Tr}\left[\sqrt{\left(\partial_{t} \varrho\right)^{2}}\right]\right)^{2}$. Using Eqs. (9) and (14) for $\partial_{t} \varrho$ and the triangle inequality, one obtains $\left\|\partial_{t} \varrho\right\| \leqslant\left\|\left[H_{\mathrm{CD}}, \varrho\right]\right\|+\|\{\Gamma, \varrho\}\|$ for the gain-loss equation and $\left\|\partial_{t} \varrho\right\| \leqslant\left\|\left[H_{\mathrm{CD}}, \varrho\right]\right\|+\left\|\mathcal{D}_{\mathrm{CD}}\right\|$ for the Lindblad-like equation. In all of these bounds, both the CD Hamiltonian and dissipator set the speed of evolution.

\section{Examples}

\subsection{Strokes for a two-level system}

Consider a two-level system described by a timedependent Hamiltonian

$$
H_{0}(t)=\frac{1}{2}\left(\Delta(t) \sigma_{z}+\Omega(t) \sigma_{x}\right)
$$

where $\sigma_{z}$ and $\sigma_{x}$ are the Pauli matrices. The instantaneous eigenstates read $E_{ \pm}(t)=$ $\pm \sqrt{\Omega^{2}(t)+\Delta^{2}(t)} / 2= \pm|\Omega(t)| /(2 \sin \theta(t))$, where $\theta(t)=\arctan (\Omega(t) / \Delta(t))$ and the corresponding eigenstates are

$$
\begin{aligned}
& \left|+{ }_{t}\right\rangle=\cos (\theta(t) / 2)|0\rangle+\sin (\theta(t) / 2)|1\rangle, \\
& \left|-{ }_{t}\right\rangle=\sin (\theta(t) / 2)|0\rangle-\cos (\theta(t) / 2)|1\rangle,
\end{aligned}
$$

with $\sigma_{z}|0\rangle=|0\rangle$ and $\sigma_{z}|1\rangle=-|1\rangle$. We consider the system to be described by the time-dependent mixed state $\varrho(t)=\sum_{\alpha= \pm} \lambda_{\alpha}(t)\left|\alpha_{t}\right\rangle\left\langle\alpha_{t}\right|$. Thus, the target trajectory $\varrho$ is already diagonal in the eigenbasis of the uncontrolled system Hamiltonian $H_{0}(t)$. The auxiliary control term required to guide the dynamics is known to be of the form $[5,6,48]$

$$
H_{1}=\frac{1}{2} \frac{\partial_{t} \Delta \Omega-\partial_{t} \Omega \Delta}{\Omega^{2}+\Delta^{2}} \sigma_{y},
$$

so that the full dynamics is generated by $H_{\mathrm{CD}}=H_{0}+$ $H_{1}$. The dynamics is open when the eigenvalues $\lambda_{ \pm}$ are time-dependent.

The first approach we have introduced relies on the presence of gain and loss, for which the dynamics is generally no longer trace-preserving, i.e., $\lambda_{-}+\lambda_{+}$is time-dependent and different from unity. Such evolution is generated by the non-Hermitian Hamiltonian $H=H_{\mathrm{CD}}-i \Gamma$, where

$$
\Gamma=\frac{\partial_{t} \lambda_{+}}{2}\left(\frac{1}{\lambda_{-}}\left|-{ }_{t}\right\rangle\left\langle-{ }_{t}\left|-\frac{1}{\lambda_{+}}\right|+{ }_{t}\right\rangle\left\langle{ }_{t}\right|\right) .
$$

Under balanced gain and loss, the trace-preserving property is restored by the nonlinear equation (11) with this choice of $\Gamma$.

Alternatively, STA in an open two-level system can be associated with a Lindblad-like master equation with the Lindblad operators

$$
L_{+-}(t)=\left|{ }_{t}\right\rangle\left\langle-{ }_{t}\right|, \quad L_{-+}(t)=L_{+-}^{\dagger}(t) .
$$

The rates are given by $\gamma_{+-}(t)=\frac{\partial_{t} \lambda_{+}}{2 \lambda_{-}}$and $\gamma_{-+}(t)=$ $\frac{\partial_{t} \lambda_{-}}{2 \lambda_{+}}$.

Assume that the system is initially prepared in a thermal state at inverse temperature $\beta(0)$, $\varrho(0)=\sum_{\alpha= \pm} \lambda_{\alpha}(0)\left|\alpha_{0}\right\rangle\left\langle\alpha_{0}\right|$, where $\lambda_{\alpha}=$ $e^{-\beta(0) E_{\alpha}(0)} / Z(0)$ with $Z(0)=e^{-\beta(0) E_{-}(0)}+$ $e^{-\beta(0) E_{+}(0)}$. We focus on description of thermodynamic protocols for which the target trajectory $\varrho(t)$ is an instantaneous thermal state with inverse temperature $\beta(t)$, i.e.,

$$
\varrho(t)=\sum_{\alpha= \pm} \frac{e^{\alpha \frac{\beta(t)}{2} \sqrt{\Omega^{2}(t)+\Delta^{2}(t)}}}{2 \cosh \left[\frac{\beta(t)}{2} \sqrt{\Omega^{2}(t)+\Delta^{2}(t)}\right]}\left|\alpha_{t}\right\rangle\left\langle\alpha_{t}\right| .
$$



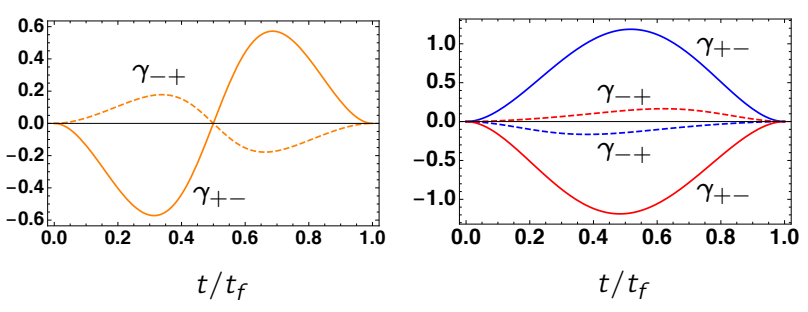

Figure 1: Left: Time-dependence of the rates in an isothermal process at inverse temperature $\beta=1$, keeping $\Omega$ constant with initial $\Delta(0)=1$ and final $\Delta\left(t_{f}\right)=$ -1 . Right: Time-dependence of the rates for the superadiabatic cooling (blue) and heating (red) of a twolevel system. Taking $\Delta=\Omega$ as the unit of frequency, the process corresponds to cooling a thermal state from $\beta(0)=1$ to $\beta\left(t_{f}\right)=2$ and heating a thermal state from $\beta(0)=2$ to $\beta\left(t_{f}\right)=1$.

One can engineer different processes of interest which are of this type. For example, in a superadiabatic isothermal stroke, the state is always in a thermal form at a given reference inverse temperature $\beta(t)=\beta(0)$, regardless of the rate at which $H_{0}(t)$ is driven. Nonadiabatic excitations are cancelled by the auxiliary term $H_{1}$ in Eq. (22), while the thermal form of $\lambda_{ \pm}(t)$ is guaranteed by the Lindblad operators and rates. For arbitrary $\Delta(t)$ and $\Omega(t)$, they read

$$
\begin{aligned}
L_{+-} & =|+\rangle\left\langle-\left|, \quad L_{-+}=\right|-\right\rangle\langle+|, \\
\gamma_{\alpha \alpha^{\prime}} & =\frac{\alpha \beta}{2} \frac{\Delta \partial_{t} \Delta+\Omega \partial_{t} \Omega}{\sqrt{\Delta^{2}+\Omega^{2}}}\left(e^{\alpha^{\prime} \beta \sqrt{\Delta^{2}+\Omega^{2}}}+1\right)^{-1},
\end{aligned}
$$

where $\alpha, \alpha^{\prime} \in\{ \pm\}$ and $\alpha \neq \alpha^{\prime}$.

A typical modulation in time is shown in Fig. 1 for a two-level system to evolve along STA for an isothermal stroke induced by driving of $\Delta(t)$ while keeping $\Omega$ constant. Specifically, $\Delta(t)$ is chosen as a fifth-order polynomial in time interpolating between the initial and final values. The rates have opposite signs, vanish identically at the avoided crossing, and flip signs during the subsequent evolution.

It is possible to look as well for cooling and heating protocols characterized by a time-dependent inverse temperature $\beta(t)$ keeping $H_{0}$ constant, as required, e.g., in a quantum Otto cycle. In such a case, $H_{1}$ vanishes, and the cooling and heating strokes are implemented by time-independent Lindblad operators with time-dependent rates,

$$
\gamma_{\alpha \alpha^{\prime}}(t)=\frac{\alpha \partial_{t} \beta(t)}{2} \frac{\sqrt{\Delta^{2}+\Omega^{2}}}{e^{-\alpha^{\prime} \sqrt{\Delta^{2}+\Omega^{2}} \beta(t)}+1},
$$

where $\alpha, \alpha^{\prime} \in\{ \pm\}$ and $\alpha \neq \alpha^{\prime}$. The timedependence of the rates is explicitly illustrated for both cooling and heating processes in Fig. 1, for constant values of $\Delta$ and $\Omega$, and $\beta(t)$ interpolating between $\beta(0)$ and $\beta\left(t_{f}\right)$ again as a fifth-order polynomial. The non-Markovian character of the evolution is manifest given the time-dependence of the Lindblad operators and the opposite sign of the corresponding rates.

Beyond these two prominent examples, more general strokes can be considered. The required Lindblad operators in the most general setting are provided in the appendixes. We also note that in all cases the corresponding operator $\Gamma$ associated with gain and loss can be conveniently expressed in terms of the rates as

$$
\Gamma(t)=\gamma_{-+}(t)\left|+{ }_{t}\right\rangle\left\langle{ }_{t}\left|-\gamma_{+-}(t)\right|-t\right\rangle\left\langle-{ }_{t}\right| .
$$

In the following, we consider another example in which the real physical dynamics of the system keeps its state always in the Gibbsian form with a timedependent temperature.

\subsection{STA for equilibration of a thermalizing atom}

Consider a two-level atom in a thermal bosonic bath at inverse temperature $\beta_{B}(0)$. The dynamics of the atom under some conditions can be described by [5759]

$$
\begin{aligned}
& \partial_{t} \varrho_{S}=-i\left[H_{S}, \varrho_{S}\right] \\
& +\sum_{j, k \mid(j \neq k)} \gamma_{j k}\left(L_{j k} \varrho_{S} L_{j k}^{\dagger}-\frac{1}{2}\left\{L_{j k}^{\dagger} L_{j k}, \varrho_{S}\right\}\right),
\end{aligned}
$$

where $j, k \in\{0,1\}$, and

$H_{S}=\frac{\omega_{0}}{2} \sigma_{z}, L_{10}=|1\rangle\left\langle 0\left|=\sigma_{-}, L_{01}=\right| 0\right\rangle\langle 1|=\sigma_{+}$,

$\gamma_{10}=\gamma\left(\bar{n}\left(\omega_{0}, \beta_{B}(0)\right)+1\right), \quad \gamma_{01}=\gamma \bar{n}$.

Here, $\bar{n}\left(\omega_{0}, \beta_{B}(0)\right)=\left(\mathrm{e}^{\beta_{B} \omega_{0}}-1\right)^{-1}$ is the mean boson number in a mode with frequency $\omega_{0}$, and $\gamma$ is a time-independent constant indicating the strength of the coupling between the atom and the thermal bath.

If the atom is initially in a thermal state $\varrho_{S}(0)=$ $e^{-\beta_{S}(0) H_{S}} / Z_{S}(0)$, its instantaneous state is obtained by solving the above master equation, which gives a Gibbsian thermal state $\varrho_{S}(t)=e^{-\beta_{S}(t) H_{S}} / Z_{S}(t)$, with

$$
\beta_{S}(t)=\frac{-1}{\omega_{0}} \log \frac{1-\mathrm{e}^{-\tilde{\gamma} t} \tanh \Theta_{S}+\left(\mathrm{e}^{-\tilde{\gamma} t}-1\right) \tanh \Theta_{B}}{1+\mathrm{e}^{-\tilde{\gamma} t} \tanh \Theta_{S}-\left(\mathrm{e}^{-\tilde{\gamma} t}-1\right) \tanh \Theta_{B}} .
$$

Here $\Theta_{k}=\omega_{0} \beta_{k}(0) / 2(k \in\{S, B\}), \tilde{\gamma}=$ $\gamma \operatorname{coth} \Theta_{B}$, and $Z_{S}(t)=\operatorname{Tr}\left[e^{-\beta_{S}(t) H_{S}}\right]$. 

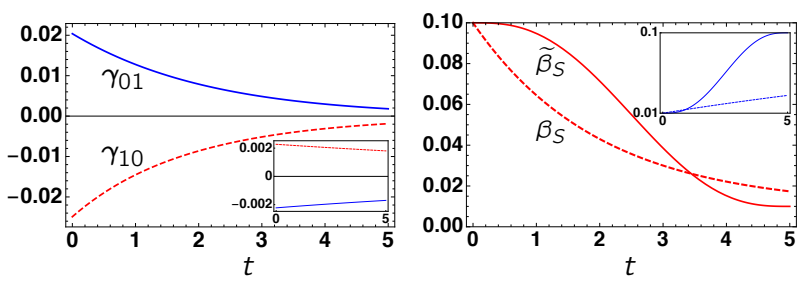

Figure 2: Left: Time dependence of the rates $\gamma_{10}$ (red, dashed) and $\gamma_{01}$ (blue, solid) in Eq. (14) for a thermalizing atom in the case of heating, when $\beta_{S}=0.1$, $\beta_{B}=0.01, \omega_{0}=2$, and $\gamma=0.005$. The inset corresponds to the cooling case, with $\beta_{S} \leftrightarrow \beta_{B}$. With these parameters, the rates for the Lindblad master equation of the subsystem in Eqs. (29) and (30) become constant; $\gamma_{01}=4.95$ and $\gamma_{10}=5.05$. Right: Inverse temperature of the system for the Lindblad master equation (dashed) and for the STA (solid). The dependence in time is shown for the case of heating (see inset for the cooling case) with the same set of parameters as in the left panel.

Equation (14) suggests another dynamical equation realizing the same trajectory $\varrho_{S}(t)$. Since $H_{0}=$ $H_{S}$ is time-independent, $H_{1}$ will be zero as well. The Lindblad operators are given in terms of the eigenstates of $H_{S}$ as $L_{m n}=|m\rangle\langle n|$ where $m, n \in\{0,1\}$, and the rates are obtained from Eq. (13b) by considering that $\lambda_{0}=e^{-\beta_{S}(t) \omega_{0} / 2} / Z_{S}(t)$ and $\lambda_{1}=$ $e^{\beta_{S}(t) \omega_{0} / 2} / Z_{S}(t)$ can also be identified simply as $\varrho_{00}$ and $\varrho_{11}$, respectively (see the appendixes). While the Lindblad operators here are equal to those in Eq. (29), the rates in the Markovian master equation (29) are positive constants. By contrast, the rates in Eq. (14) are time-dependent and negative for some time intervals, as illustrated in Fig. 2. Nonetheless, in both cases equilibration with the bath at temperature $\beta_{B}$ takes infinite time.

A STA in finite time $t_{f}$ can be associated with a trajectory $\tilde{\varrho}_{S}(t)=e^{-\tilde{\beta}_{S}(t) H_{S}} / Z_{S}(t)$ and a modified inverse temperature $\tilde{\beta}_{S}(t)$ satisfying $\tilde{\beta}_{S}\left(t_{f}\right)=\beta_{B}$. Using Eq. (14), the Lindblad operators remain unchanged, as in Eq. (30), whereas the rates are obtained from Eq. (13b) as $\gamma_{01}=-\left(\omega_{0} / 4\right) \partial_{t} \tilde{\beta}_{S}(t) e^{-\tilde{\beta}_{S}(t) \omega_{0}}$ and $\gamma_{10}=$ $\left(\omega_{0} / 4\right) \partial_{t} \tilde{\beta}_{S}(t) e^{\tilde{\beta}_{S}(t) \omega_{0}}$. In Fig. 2, the right panel shows the temperature for a typical function as $\tilde{\beta}_{S}(t)$ such that at $t_{f}=5$ the system state thermalizes, i.e., $\tilde{\varrho}_{S}\left(t_{f}\right)=e^{-\beta_{B} H_{S}} / \operatorname{Tr}\left[e^{-\beta_{B} H_{S}}\right]$.

\subsection{Fast thermalization of a quantum oscillator}

We next consider the fast thermalization of a quantum oscillator using the general scheme presented in
Sec. 2. This illustrate an application of the proposed scheme for an infinite rank density matrix, that can be implemented with current technology. Alternative approaches for the fast thermalization of an oscillator have been recently presented in Ref. [43, 44].

Consider the time-dependent Hamiltonian $H_{0}=$ $\frac{\hat{p}^{2}}{2 m}+\frac{1}{2} m \omega_{t}^{2} \hat{x}^{2}$ with instantaneous thermal state $\varrho(t)=e^{-\beta_{t} H_{0}} / \operatorname{Tr}\left[e^{-\beta_{t} H_{0}}\right]$. In the basis of the instantaneous Fock states $\left|n_{t}\right\rangle$, the thermal state is diagonal, $\varrho(t)=\sum_{n} \lambda_{n}(t)\left|n_{t}\right\rangle\left\langle n_{t}\right|$, with probabilities $\lambda_{n}(t)=u_{t}^{n}\left(1-u_{t}\right)^{-1}$ that are generally timedependent due to the modulation of the frequency and temperature, where $u_{t}=e^{-\beta_{t} \hbar \omega_{t}}$. The thermal state evolves according to Eq. (7), where the commutator $\left[H_{0}, \varrho(t)\right]=0$ and the counterdiabatic Hamiltonian term $H_{1}$ is given by [60-62]

$$
H_{1}=-\frac{\dot{\omega}_{t}}{4 \omega_{t}}\{\hat{x}, \hat{p}\} .
$$

This term can in principle be engineered in a trapped ion as suggested in Ref. [9]. We show below a scheme for implementing in the laboratory the unitarily equivalent trajectory $\tilde{\varrho}(t)=U_{x} \varrho(t) U_{x}^{\dagger}$, where

$$
U_{x}=e^{i \frac{m}{2 \hbar} \alpha_{t} \hat{x}^{2}},
$$

and $\alpha_{t}$ is a frequency to be determined. Such trajectory maps an initial thermal state into a final thermal state of different temperature provided $\alpha_{t}$ vanishes at the beginning and end of the protocol. Direct computation of its time derivative gives $\partial_{t} \tilde{\varrho}=$ $\frac{i}{\hbar}\left[\frac{m}{2} \dot{\alpha}_{t} \hat{x}^{2}, \tilde{\varrho}\right]+U_{x}\left(\partial_{t} \varrho\right) U_{x}^{\dagger}$, which admits a form similar to Eq. (7), i.e,

$$
\partial_{t} \tilde{\varrho}=-\frac{i}{\hbar}\left[\tilde{H}_{\mathrm{CD}}, \tilde{\varrho}\right]+\tilde{\mathcal{D}}_{\mathrm{CD}}(\tilde{\varrho}),
$$

where the counterdiabatic Hamiltonian in the rotating frame reads

$$
\tilde{H}_{\mathrm{CD}}=i \hbar \dot{U}_{x} U_{x}^{\dagger}+U_{x}\left(H_{0}+H_{1}\right) U_{x}^{\dagger},
$$

and the dissipator is given by

$$
\tilde{\mathcal{D}}_{\mathrm{CD}}(\tilde{\varrho})=\sum_{n} \dot{\lambda}_{n}(t) U_{x}\left|n_{t}\right\rangle\left\langle n_{t}\right| U_{x}^{\dagger} .
$$

By explicit computation, the counterdiabatic Hamiltonian Eq. (35) can be recast as

$$
\tilde{H}_{\mathrm{CD}}=\frac{\hat{p}^{2}}{2 m}+\frac{1}{2} m \tilde{\omega}_{t}^{2} \hat{x}^{2}-\left(\frac{\alpha_{t}}{2}+\frac{\dot{\omega}_{t}}{4 \omega_{t}}\right)\{\hat{x}, \hat{p}\},
$$

with time-dependent frequency

$$
\tilde{\omega}_{t}^{2}=\omega_{t}^{2}+\alpha_{t}^{2}+\alpha_{t} \frac{\dot{\omega}_{t}}{\omega_{t}}-\dot{\alpha}_{t}
$$


It proves convenient to define $\alpha_{t}=\Omega_{t}-\dot{\omega}_{t} /\left(2 \omega_{t}\right)$, so that

$$
\tilde{H}_{\mathrm{CD}}=\frac{\hat{p}^{2}}{2 m}+\frac{1}{2} m \tilde{\omega}_{t}^{2} \hat{x}^{2}-\frac{\Omega_{t}}{2}\{\hat{x}, \hat{p}\} .
$$

As shown in App. C, by further choosing

$$
\Omega_{t}=-\frac{1}{2} \frac{\dot{\omega}_{t}}{\omega_{t}}+\frac{\dot{u}_{t}}{1-u_{t}^{2}}
$$

the dissipator in the rotating frame equals

$$
\begin{aligned}
\tilde{\mathcal{D}}_{\mathrm{CD}}(\tilde{\varrho})= & \frac{1}{i \hbar}\left[\frac{\Omega_{t}}{2}\{\hat{x}, \hat{p}\}-m \alpha_{t} \Omega_{t} \hat{x}^{2}, \tilde{\varrho}\right] \\
& -\gamma_{t}[\hat{x},[\hat{x}, \tilde{\varrho}]],
\end{aligned}
$$

with a time-dependent dephasing strength

$$
\gamma_{t}=\frac{m \omega_{t}}{\hbar} \frac{\dot{u}_{t}}{\left(1-u_{t}\right)^{2}}
$$

Combining the explicit forms of $\tilde{H}_{\mathrm{CD}}$ and $\tilde{\mathcal{D}}_{\mathrm{CD}}(\tilde{\varrho})$ in Eq. (34) results in the master equation of a timedependent quantum oscillator subject to dephasing in the coordinate representation, i.e.,

$$
\partial_{t} \tilde{\varrho}=\frac{1}{i \hbar}\left[\frac{\hat{p}^{2}}{2 m}+\frac{1}{2} m \tilde{\omega}_{\mathrm{CD}}^{2} \hat{x}^{2}, \tilde{\varrho}\right]-\gamma_{t}[\hat{x},[\hat{x}, \tilde{\varrho}]] .
$$

where

$$
\begin{aligned}
\tilde{\omega}_{\mathrm{CD}}^{2} & =\omega_{t}^{2}-\alpha_{t}^{2}-\dot{\alpha}_{t} \\
& =\left[\omega_{t}^{2}-\frac{3}{4}\left(\frac{\dot{\omega}_{t}}{\omega_{t}}\right)^{2}+\frac{\ddot{\omega}_{t}}{2 \omega_{t}}\right]-\Omega_{t}^{2}-\dot{\Omega}_{t}+\Omega_{t} \frac{\dot{\omega}_{t}}{\omega_{t}},
\end{aligned}
$$

and $\gamma_{t}$ is given by Eq. (42) and $\Omega_{t}$ by Eq. (40). The case of unitary dynamics in which the eigenvalues $\left\{\lambda_{n}\right\}$ are constant corresponds to $\Omega_{t}=0$, i.e., $\alpha_{t}=-\dot{\omega}_{t} /\left(2 \omega_{t}\right)$. The first term in square brackets on the right-hand side (RHS) of Eq. (44) is indeed that used for the (local) counterdiabatic driving of a driven oscillator in the absence of coupling to a bath $[9,62,63]$. The dynamics described by Eq. (43) generalizes the case of unitary evolution to account for the controlled driving of an open quantum oscillator (i.e., when the eigenvalues $\left\{\lambda_{n}\right\}$ of the density matrix are time-dependent) from an initial thermal state to a final thermal state in arbitrary time. The implementation of a STA by counterdiabatic driving for the fast thermalization of a quantum oscillator is achieved by a simultaneous modulation of the driving frequency and the dephasing strength. The dynamics associated with Eq. (43) can be readily implemented in the laboratory. It requires the control of the trap frequency and dephasing strength. The latter can be engineered for $\gamma_{t}>0$ using noise as a resource via stochastic parametric driving, or through continuous quantum measurements, as recently proposed in Ref. [44]. While the counterdiabatic driving protocol derived here requires similar experimental resources to the ones for STA based on reverse-engineering of the dynamics [44], the time modulations of the driving frequency and the dephasing strength need not be equal, and generally differ, between the two protocols. In addition, their experimental implementation is at reach with current technology in trapped ions $[9,64]$ and ultracold gases [10].

To illustrate a specific protocol we consider a reference trajectory $\varrho(t)$ describing the evolution from an initial thermal state of frequency $\omega_{0}$ at inverse temperature $\beta_{0}$ to a final thermal state with frequency $\omega\left(t_{f}\right)=\omega_{f}$ and inverse temperature $\beta\left(t_{f}\right)=\beta_{f}$. For instance, $\varrho(t)$ can be specified by choosing the interpolating ansatze

$$
\begin{aligned}
\omega_{t} & =\omega_{0}+\left(\omega_{f}-\omega_{0}\right)\left[10 s^{3}-15 s^{4}+6 s^{5}\right], \\
\beta_{t} & =\beta_{0}+\left(\beta_{f}-\beta_{0}\right)\left[10 s^{3}-15 s^{4}+6 s^{5}\right],
\end{aligned}
$$

with $s=t / t_{f}$, where $t_{f}$ is the duration of the process. The polynomial functions are monotonic as a function of time. The required experimental controls to implement the unitarily equivalent trajectory $\tilde{\varrho}(t)$ are $\tilde{\omega}_{\mathrm{CD}}$ in Eq. (44) and $\gamma_{t}$ in Eq. (42) with $u_{t}=e^{-\beta_{t} \hbar \omega_{t}}$, shown in Fig. 3. Specifically, a heating stroke involving a trap compression shows that the required counterdiabatic modulation of the trapping frequency exhibits a nonmonotonic behavior involving of sequence of tight compressions and decompressions, overshooting the reference modulation. Along the process, the dephasing strength takes predominantly positive values, thus suppressing coherences in the position eigenbasis. counterdiabatic cooling strokes are more challenging to implement than counterdiabatic heating strokes. First, the dephasing strength takes negative values throughout the cooling stroke, enhancing coherences in the position representation. Second, the square frequency of the trap exhibits as well a nonmonotonic behavior characterized, acquiring transient negative values associated with a purely imaginary frequency, e.g., the inversion of the trap into an anti-trap. Such inversions are also common to counterdiabatic driving for unitary processes whenever the duration of the process is comparable to $\omega_{0}^{-1}$ [4]. While the inversion of the trap is not free from technical difficulties, its realization has been facilitated by the development of the painting potential 

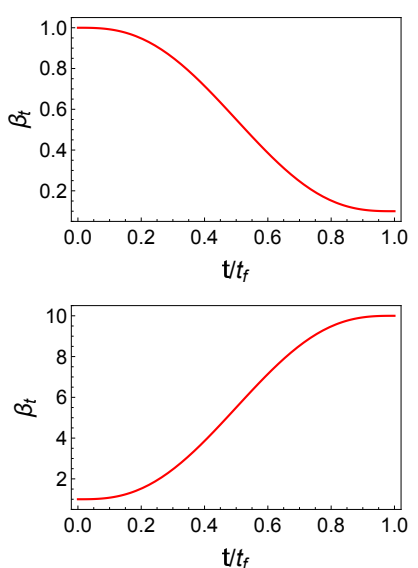
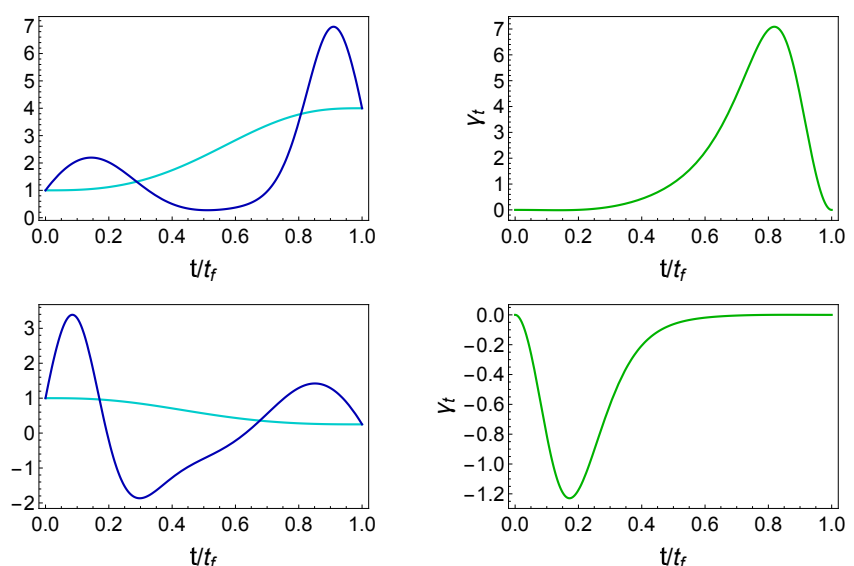

Figure 3: Counterdiabatic driving of an open driven quantum quantum oscillator. Heating stroke (top): Left: Modulation of the inverse-temperature with $\beta_{f}=\beta_{0} / 10$. Center: Monotonic reference modulation of the trapping square frequency $\omega_{t}^{2} / \omega_{0}^{2}$ compared with the nonmonotonic counterdiabatic modulation $\tilde{\omega}_{\mathrm{CD}}^{2} / \omega_{0}^{2}$, with $\omega_{f}=2 \omega_{0}$. Right: Time-dependent dephasing strength in units of $m \omega_{0} / \hbar$. The bottom row shows the engineering of a cooling stroke with $\omega_{f}=\omega_{0} / 2$ and $\beta_{f}=10 \beta_{0}$. Negative values of $\tilde{\omega}_{\mathrm{CD}}^{2} / \omega_{0}^{2}$ are associated with trap inversion.

technique and the use of digital micromirror devices [65] as suggested in Ref. [66].

\section{Summary and conclusions}

We have introduced a universal scheme to design shortcuts to adiabaticity in open quantum systems, interacting with an environment. This scheme provides the generalization of counterdiabatic driving [5, 6], also known as transitionless quantum driving [48], to open quantum systems. It is based on first prescribing a target trajectory for the evolution of the system, and then determining the required auxiliary Hamiltonian terms and dissipators that generate it.

The resulting dynamics admits different physical realizations. It can be associated with a driven system in the presence of balanced gain and loss, a scenario that occurs naturally, e.g., in $\mathcal{P} \mathcal{T}$-symmetric quantum mechanics. Alternatively, it can be implemented via a non-Markovian evolution in which the equation governing the dynamics takes a generalized Lindblad-like form. The latter is readily accessible in a variety of platforms-including trapped ions, Rydberg atoms, and superconducting qubits, among other examples-by using, e.g., digital quantum simulation techniques [67-71]. Our formalism thus enables to engineer superadiabatic open processes to speed up, i.e., heating, cooling, and isothermal strokes.

We have applied this framework to the engineering of strokes in an open two-level system. In addition, we have provided an experimentally-friendly protocol for the the controlled thermalization of a driven quantum oscillator, that can be implemented with current technology in trapped ions and ultracold gases. The framework introduced here should find broad applications in quantum thermodynamics, and more generally, in quantum technologies requiring the fast control of an open system embedded in an environment.

Acknowledgements.-We would like to thank Tapio Ala-Nissila, Léonce Dupays, and Jack J. Mayo for comments on the manuscript. This work is supported by ID2019-109007GA-I00. Further support by the Academy of Finland's Center of Excellence program QTF Project 312057 (to S.A.) is acknowledged. A.T.R. also acknowledges support by the QTF, Aalto University's AScI Visiting Professor Fund, and Sharif University of Technology's Office of Vice President for Research and Technology.

\section{A Lindblad-like master equation}

In this section, we verify that the dissipator

$$
\mathcal{D}(\varrho)=\sum_{m n} \gamma_{m n}\left(L_{m n} \varrho L_{m n}^{\dagger}-\frac{1}{2}\left\{L_{m n}^{\dagger} L_{m n}, \varrho\right\}\right),
$$

with the choice of the time-dependent Lindblad operators and rates given in the main text, satisfies the identity

$$
\mathcal{D}(\varrho)=\sum_{m} \partial_{t} \lambda_{m}(t)\left|m_{t}\right\rangle\left\langle m_{t}\right| .
$$

Employing the explicit form of $L_{m n}$ in Eq. (47) one finds

$$
\mathcal{D}(\varrho)=\sum_{m n} \frac{\partial_{t} \lambda_{m}(t)}{r}\left(\left|m_{t}\right\rangle\left\langle m_{t}|-| n_{t}\right\rangle\left\langle n_{t}\right|\right) \text {.( }
$$


Noting that $\sum_{n=1}^{r} 1=r=\operatorname{rank}(\varrho)$ and $\sum_{m=1}^{r} \partial_{t} \lambda_{m}(t)=\partial_{t} \operatorname{Tr}[\varrho]$, it follows that

$$
\begin{aligned}
\mathcal{D}(\varrho)= & \sum_{m} \partial_{t} \lambda_{m}(t)\left|m_{t}\right\rangle\left\langle m_{t}\right| \\
& -(1 / r) \partial_{t} \operatorname{Tr}[\varrho] \sum_{n}\left|n_{t}\right\rangle\left\langle n_{t}\right| .
\end{aligned}
$$

As the second term on the RHS vanishes identically for a norm-preserving evolution, this completes the proof of Eq. (48).

\section{B Lindblad operators for arbitrary strokes in two-level systems}

Consider the trajectory described by the instantaneous thermal state of a two-level system

$$
\varrho(t)=\sum_{\alpha= \pm} \frac{e^{\alpha \frac{\beta}{2} \sqrt{\Omega^{2}+\Delta^{2}}}}{2 \cosh \left[\frac{\beta}{2} \sqrt{\Omega^{2}+\Delta^{2}}\right]}\left|\alpha_{t}\right\rangle\left\langle\alpha_{t}\right|,
$$

where $\beta, \Delta$, and $\Omega$ are time-dependent. The Lindblad operators are $L_{+-}=|+\rangle\langle-|$and $L_{-+}=|-\rangle\langle+|$, as in Eq. (25) in the main text, with rates

$$
\begin{aligned}
\gamma_{+-}(t) & =\frac{\Delta^{2} \partial_{t} \beta+\Omega\left(\Omega \partial_{t} \beta+\beta \partial_{t} \Omega\right)+\beta \Delta \partial_{t} \Delta}{2 \sqrt{\Delta^{2}+\Omega^{2}}\left(e^{-\beta \sqrt{\Delta^{2}+\Omega^{2}}}+1\right)}, \\
\gamma_{-+}(t) & =-\frac{\Delta^{2} \partial_{t} \beta+\Omega\left(\Omega \partial_{t} \beta+\beta \partial_{t} \Omega\right)+\beta \Delta \partial_{t} \Delta}{2 \sqrt{\Delta^{2}+\Omega^{2}}\left(e^{\beta \sqrt{\Delta^{2}+\Omega^{2}}}+1\right)} .
\end{aligned}
$$

\section{Thermalization of a quantum oscil- lator}

We provide details to establish the equivalence of the different master equations for the fast thermalization of a quantum oscillator. To do this, we use the coordinate representation. The thermal state of a harmonic oscillator is known to be described by a Gaussian density matrix,

$$
\varrho\left(x, x^{\prime}, t\right)=\left\langle x|\varrho(t)| x^{\prime}\right\rangle=N_{t} e^{-A_{t}\left(x^{2}+x^{2}\right)-2 C_{t} x x^{\prime}},
$$

with normalization constant $N_{t}=\sqrt{2\left(A_{t}+C_{t}\right) / \pi}$. The real parameters $A_{t}=k_{t}^{2}\left(1+u_{t}^{2}\right) /\left(2\left(1-u_{t}^{2}\right)\right)$ and $C_{t}=-k_{t}^{2} u_{t} /\left(1-u_{t}^{2}\right)$ follow from the inverse length $k_{t}=\sqrt{m \omega_{t} / \hbar}$ and the normalization factor $N_{t}=$ $k_{t} \sqrt{\left(1-u_{t}\right) /\left(\pi\left(1+u_{t}\right)\right)}$. This gives the coordinate representation of the dissipator (34) as

$$
\begin{aligned}
& \left\langle x\left|\tilde{\mathcal{D}}_{\mathrm{CD}}(\tilde{\varrho})\right| x^{\prime}\right\rangle=\frac{\partial \varrho\left(x, x^{\prime}, t\right)}{\partial t} e^{i \frac{m \alpha_{t}}{2 \hbar}\left(x^{2}-x^{\prime 2}\right)} \\
& =\left(\frac{\dot{N}_{t}}{N_{t}}-\dot{A}_{t}\left(x^{2}+x^{\prime 2}\right)-2 \dot{C}_{t} x x^{\prime}\right) \tilde{\varrho}\left(x, x^{\prime}, t\right) .
\end{aligned}
$$

Given the explicit form of $N_{t}$, choosing $\Omega_{t}=$ $-\frac{\dot{N}_{t}}{N_{t}}$, as in Eq. (40), leads to $\dot{A}_{t}+2 \Omega_{t} A_{t}=-\dot{C}_{t}-$ $2 \Omega_{t} C_{t} \equiv \gamma_{t}$. The latter corresponds to a dephasing strength, and allows recasting the dissipator as

$$
\begin{aligned}
\left\langle x\left|\tilde{\mathcal{D}}_{\mathrm{CD}}(\tilde{\varrho})\right| x^{\prime}\right\rangle=[ & \Omega_{t}\left(2 A\left(x^{2}+x^{\prime 2}\right)+4 C x x^{\prime}-1\right) \\
& \left.-\gamma_{t}\left(x-x^{\prime}\right)^{2}\right] \tilde{\varrho}\left(x, x^{\prime}, t\right) .
\end{aligned}
$$

We wish to rewrite this last expression in operator form. To that end we note that $\left[H_{0}, \varrho(t)\right]=0$ and thus $\left[U H_{0} U^{\dagger}, \tilde{\varrho}(t)\right]=0$, whence it follows that

$$
[\{\hat{x}, \hat{p}\}, \tilde{\varrho}]=\frac{2}{\alpha}\left[\frac{\hat{p}^{2}}{2 m}+\frac{1}{2} m\left(\omega_{t}^{2}+\alpha_{t}^{2}\right) \hat{x}^{2}, \tilde{\varrho}\right] .
$$

Explicit computation using the coordinate representation of the trajectory, $\tilde{\varrho}\left(x, x^{\prime}, t\right)=$ $\varrho\left(x, x^{\prime}, t\right) e^{i \frac{m \alpha_{t}}{2 \hbar}\left(x^{2}-x^{\prime 2}\right)}$, yields

$$
\begin{aligned}
& \left\langle x|[\{\hat{x}, \hat{p}\}, \tilde{\varrho}(t)]| x^{\prime}\right\rangle=2 m \alpha_{t}\left(x^{2}-x^{\prime 2}\right) \tilde{\varrho}\left(x, x^{\prime}, t\right) \\
& \quad+2 i \hbar\left(2 A\left(x^{2}+x^{\prime 2}\right)+4 C x x^{\prime}-1\right) \varrho\left(x, x^{\prime}, t\right) .
\end{aligned}
$$

As a result, the dissipator admits the operator form of the dissipator given in the main text, Eq. (41).

\section{References}

[1] Erik Torrontegui, Sara Ibáñez, Sofia MartínezGaraot, Michele Modugno, Adolfo del Campo, David Guéry-Odelin, Andreas Ruschhaupt, Xi Chen, and Juan Gonzalo Muga. Chapter 2: Shortcuts to adiabaticity. In Ennio Arimondo, Paul R. Berman, and Chun C. Lin, editors, Advances in Atomic, Molecular, and Optical Physics, Vol. 62, pp. 117. Academic Press, 2013. DOI: 10.1016/B978-0-12-4080904.00002-5.

[2] Adolfo del Campo and Kihwan Kim. Focus on shortcuts to adiabaticity. New J. Phys., 21:050201, 2019. DOI: 10.1088/13672630/ab1437.

[3] D. Guéry-Odelin, A. Ruschhaupt, A. Kiely, E. Torrontegui, S. Martínez-Garaot, and J. G. Muga. Shortcuts to adiabaticity: Concepts, methods, and applications. Rev. Mod. Phys., 91:045001, 2019. DOI: 10.1103/RevModPhys.91.045001.

[4] Xi Chen, A. Ruschhaupt, S. Schmidt, A. del Campo, D. Guéry-Odelin, and J. G. Muga. 
Fast optimal frictionless atom cooling in harmonic traps: Shortcut to adiabaticity. Phys. Rev. Lett., 104:063002, 2010. DOI: 10.1103/PhysRevLett.104.063002.

[5] Mustafa Demirplak and Stuart A Rice. Adiabatic population transfer with control fields. J. Phys. Chem. A, 107:9937, 2003. DOI: 10.1021/jp030708a.

[6] Mustafa Demirplak and Stuart A Rice. Assisted adiabatic passage revisited. J. Phys. Chem. B, 109:6838, 2005. DOI: 10.1021/jp040647w.

[7] Jiawen Deng, Qing-Hai Wang, Zhihao Liu, Peter Hänggi, and Jiangbin Gong. Boosting work characteristics and overall heat-engine performance via shortcuts to adiabaticity: Quantum and classical systems. Phys. Rev. E, 88:062122, 2013. DOI: 10.1103/PhysRevE.88.062122.

[8] Adolfo del Campo, J Goold, and M Paternostro. More bang for your buck: Super-adiabatic quantum engines. Sci. Rep., 4:6208, 2014. DOI: 10.1038/srep06208.

[9] Ken Funo, Jing-Ning Zhang, Cyril Chatou, Kihwan Kim, Masahito Ueda, and Adolfo del Campo. Universal work fluctuations during shortcuts to adiabaticity by counterdiabatic driving. Phys. Rev. Lett., 118:100602, 2017. DOI: 10.1103/PhysRevLett.118.100602.

[10] J.-F. Schaff, X.-L. Song, P. Vignolo, and G. Labeyrie. Fast optimal transition between two equilibrium states. Phys. Rev. A, 82:033430, 2010. DOI: 10.1103/PhysRevA.82.033430.

[11] J.-F. Schaff, X.-L. Song, P. Capuzzi, P. Vignolo, and G. Labeyrie. Shortcut to adiabaticity for an interacting Bose-Einstein condensate. Europhys. Lett., 93:23001, 2011. DOI: 10.1209/0295-5075/93/23001.

[12] M. G. Bason, M. Viteau, N. Malossi, P. Huillery, E. Arimondo, R. Fazio, V. Giovannetti, R. Mannella, and O. Morsch. High-fidelity quantum driving. Nat. Phys., 8:147, 2012. DOI: $10.1038 /$ nphys 2170 .

[13] W. Rohringer, D. Fischer, F. Steiner, I. E. Mazets, J. Schmiedmayer, and M. Trupke. Nonequilibrium scale invariance and shortcuts to adiabaticity in a one-dimensional Bose gas. Sci. Rep., 5:9820, 2015. DOI: 10.1038/srep09820.

[14] Shujin Deng, Pengpeng Diao, Qianli Yu, Adolfo del Campo, and Haibin Wu. Shortcuts to adiabaticity in the strongly coupled regime: Nonadiabatic control of a unitary fermi gas. Phys. Rev. A, 97:013628, 2018. DOI: 10.1103/PhysRevA.97.013628.

[15] Shujin Deng, Aurélia Chenu, Pengpeng Diao, Fang Li, Shi Yu, Ivan Coulamy, Adolfo del Campo, and Haibin Wu. Superadiabatic quantum friction suppression in finite-time thermodynamics. Sci. Adv., 4:eaar5909, 2018. DOI: 10.1126/sciadv.aar5909.

[16] Pengpeng Diao, Shujin Deng, Fang Li, Shi Yu, Aurélia Chenu, Adolfo del Campo, and Haibin $\mathrm{Wu}$. Shortcuts to adiabaticity in fermi gases. New J. Phys., 20:105004, oct 2018. DOI: 10.1088/1367-2630/aae45e.

[17] Jingfu Zhang, Jeong Hyun Shim, Ingo Niemeyer, T. Taniguchi, T. Teraji, H. Abe, S. Onoda, T. Yamamoto, T. Ohshima, J. Isoya, and Dieter Suter. Experimental implementation of assisted quantum adiabatic passage in a single spin. Phys. Rev. Lett., 110:240501, 2013. DOI: 10.1103/PhysRevLett.110.240501.

[18] J. Kölbl, A. Barfuss, M. S. Kasperczyk, L. Thiel, A. A. Clerk, H. Ribeiro, and P. Maletinsky. Initialization of single spin dressed states using shortcuts to adiabaticity. Phys. Rev. Lett., 122:090502, 2019. DOI: 10.1103/PhysRevLett.122.090502.

[19] Shuoming An, Dingshun Lv, Adolfo del Campo, and Kihwan Kim. Shortcuts to adiabaticity by counterdiabatic driving for trapped-ion displacement in phase space. Nat. Commun., 7:12999, 2016. DOI: 10.1038/ncomms12999.

[20] Tenghui Wang, Zhenxing Zhang, Liang Xiang, Zhilong Jia, Peng Duan, Weizhou Cai, Zhihao Gong, Zhiwen Zong, Mengmeng Wu, Jianlan $\mathrm{Wu}$, Luyan Sun, Yi Yin, and Guoping Guo. The experimental realization of highfidelity 'shortcut-to-adiabaticity' quantum gates in a superconducting xmon qubit. New J. Phys., 20:065003, 2018. DOI: 10.1088/13672630/aac9e7.

[21] Tenghui Wang, Zhenxing Zhang, Liang Xiang, Zhilong Jia, Peng Duan, Zhiwen Zong, Zhenhai Sun, Zhangjingzi Dong, Jianlan Wu, Yi Yin, and Guoping Guo. Experimental realization of a fast controlled- $Z$ gate via a shortcut to adiabaticity. Phys. Rev. Applied, 11:034030, 2019. DOI: 10.1103/PhysRevApplied.11.034030.

[22] H.-P. Breuer and P. Petruccione. The Theory of Open Quantum Systems. Oxford University Press, Oxford, 2007. 
[23] M. B. Plenio and P. L. Knight. The quantumjump approach to dissipative dynamics in quantum optics. Rev. Mod. Phys., 70:101, 1998. DOI: 10.1103/RevModPhys.70.101.

[24] Carl M. Bender and Stefan Boettcher. Real spectra in non-Hermitian Hamiltonians having PT symmetry. Phys. Rev. Lett., 80:5243, 1998. DOI: 10.1103/PhysRevLett.80.5243.

[25] Christian E. Rüter, Konstantinos G. Makris, Ramy El-Ganainy, Demetrios N. Christodoulides, Mordechai Segev, and Detlef Kip. Observation of parity-time symmetry in optics. Nat. Phys., 6:192, 2010. DOI: 10.1038/nphys 1515 .

[26] Alois Regensburger, Christoph Bersch, Mohammad-Ali Miri, Georgy Onishchukov, Demetrios N. Christodoulides, and Ulf Peschel. Parity-time synthetic photonic lattices. Nature (London), 488:167, 2012. DOI: 10.1038/nature11298.

[27] Liang Feng, Ye-Long Xu, William S. Fegadolli, Ming-Hui Lu, José E. B. Oliveira, Vilson R. Almeida, Yan-Feng Chen, and Axel Scherer. Experimental demonstration of a unidirectional reflectionless parity-time metamaterial at optical frequencies. Nat. Mater., 12:108, 2012. DOI: 10.1038/nmat3495.

[28] Bo Peng, Sahin Kaya Özdemir, Fuchuan Lei, Faraz Monifi, Mariagiovanna Gianfreda, Gui Lu Long, Shanhui Fan, Franco Nori, Carl M. Bender, and Lan Yang. Paritytime-symmetric whispering-gallery microcavities. Nat. Phys., 10:394, 2014. DOI: 10.1038/nphys2927.

[29] Bo Zhen, Chia Wei Hsu, Yuichi Igarashi, Ling Lu, Ido Kaminer, Adi Pick, Song-Liang Chua, John D. Joannopoulos, and Marin Soljačić. Spawning rings of exceptional points out of dirac cones. Nature (London), 525:354, 2015. DOI: 10.1038/nature14889.

[30] A Ruschhaupt, Xi Chen, D Alonso, and J G Muga. Optimally robust shortcuts to population inversion in two-level quantum systems. New J. Phys., 14: 093040, 2012. DOI: 10.1088/13672630/14/9/093040.

[31] Anthony Kiely and Andreas Ruschhaupt. Inhibiting unwanted transitions in population transfer in two-and three-level quantum systems. J. Phys. B: At. Mol. Opt. Phys., 47:115501, 2014. DOI: 10.1088/09534075/47/11/115501.
[32] D. A. Lidar, I. L. Chuang, and K. B. Whaley. Decoherence-free subspaces for quantum computation. Phys. Rev. Lett., 81:2594, 1998. DOI: 10.1103/PhysRevLett.81.2594.

[33] S. L. Wu, X. L. Huang, H. Li, and X. X. Yi. Adiabatic evolution of decoherence-free subspaces and its shortcuts. Phys. Rev. A, 96:042104, 2017. DOI: 10.1103/PhysRevA.96.042104.

[34] Amikam Levy, A. Kiely, J. G. Muga, R. Kosloff, and E. Torrontegui. Noise resistant quantum control using dynamical invariants. New J. Phys., 20:025006, 2018. DOI: 10.1088/13672630/aaa9e5.

[35] A. B. Boyd, A. Patra, C. Jarzynski, and J. P. Crutchfield. Shortcuts to thermodynamic computing: The cost of fast and faithful erasure. arXiv:1812.11241, 2018. URL https://arxiv. org/abs/1812.11241.

[36] Adolfo del Campo, Aurélia Chenu, Shujin Deng, and Haibin Wu. Friction-Free Quantum Machines. In F. Binder, L. Correa, C. Gogolin, J. Anders, and G. Adesso, editors, Thermodynamics in the Quantum Regime, pp. 127. Springer International Publishing, Cham, 2018. DOI: 10.1007/978-3-319-99046-0_5.

[37] S. Ibáñez, S. Martínez-Garaot, Xi Chen, E. Torrontegui, and J. G. Muga. Shortcuts to adiabaticity for non-Hermitian systems. Phys. Rev. A, 84:023415, 2011. DOI: 10.1103/PhysRevA.84.023415.

[38] Guan-Qiang Li, Guang-De Chen, Ping Peng, and Wei Qi. Non-Hermitian shortcut to adiabaticity of two-and three-level systems with gain and loss. Eur. Phys. J. D, 71:1, 2017. DOI: 10.1140/epjd/e2016-70525-6.

[39] Ye-Hong Chen, Qi-Cheng Wu, Bi-Hua Huang, Jie Song, Yan Xia, and Shi-Biao Zheng. Improving shortcuts to non-Hermitian adiabaticity for fast population transfer in open quantum systems. Ann. Phys. (Berlin), 530: 1700247, 2018. DOI: 10.1002/andp.201700247.

[40] François Impens and David Guéry-Odelin. Fast quantum control in dissipative systems using dissipationless solutions. Sci. Rep., 9:1, 2019. DOI: 10.1038/s41598-019-39731-z.

[41] G. Vacanti, R. Fazio, S. Montangero, G. M. Palma, M. Paternostro, and V. Vedral. Transitionless quantum driving in open quantum systems. New J. Phys., 16:053017, 2014. DOI: 10.1088/1367-2630/16/5/053017.

[42] M. S. Sarandy and D. A. Lidar. Adiabatic ap- 
proximation in open quantum systems. Phys. Rev. A, 71:012331, 2005. DOI: 10.1103/PhysRevA.71.012331.

[43] Roie Dann, Ander Tobalina, and Ronnie Kosloff. Shortcut to equilibration of an open quantum system. Phys. Rev. Lett., 122:250402, 2019. DOI: 10.1103/PhysRevLett.122.250402.

[44] L. Dupays, I. L. Egusquiza, A. del Campo, and A. Chenu. Superadiabatic thermalization of a quantum oscillator by engineered dephasing. Phys. Rev. Research, 2:033178, 2020. DOI: 10.1103/PhysRevResearch.2.033178.

[45] Tamiro Villazon, Anatoli Polkovnikov, and Anushya Chandran. Swift heat transfer by fast-forward driving in open quantum systems. Phys. Rev. A, 100:012126, 2019. DOI: 10.1103/PhysRevA.100.012126.

[46] N. Pancotti, M. Scandi, M. T. Mitchison, and M. Perarnau-Llobet. Speed-ups to isothermality: Enhanced quantum heat engines through control of the system-bath coupling. arXiv:1911.12437, 2019. URL https://arxiv. org/abs/1911.12437.

[47] L. Dupays and A. Chenu. Dynamical engineering of squeezed thermal states, 2020. URL https://arxiv.org/abs/2008.03307.

[48] M. V. Berry. Transitionless quantum driving. $J$. Phys. A: Math. Theor., 42:365303, 2009. DOI: 10.1088/1751-8113/42/36/365303.

[49] Tosio Kato. On the adiabatic theorem of quantum mechanics. J. Phys. Soc. Jpn., 5:435, 1950. DOI: 10.1143/JPSJ.5.435.

[50] J. E. Avron, R. Seiler, and L. G. Yaffe. Adiabatic theorems and applications to the quantum Hall effect. Commun. Math. Phys., 110:33, 1987. DOI: 10.1007/BF01209015.

[51] Dorje C. Brody and Eva-Maria Graefe. Mixedstate evolution in the presence of gain and loss. Phys. Rev. Lett., 109:230405, 2012. DOI: 10.1103/PhysRevLett.109.230405.

[52] Jiangbin Gong and Qing-Hai Wang. Timedependent $\mathcal{P} \mathcal{T}$-symmetric quantum mechanics. J. Phys. A: Math. Theor, 46:485302, 2013. DOI: $10.1088 / 1751-8113 / 46 / 48 / 485302$.

[53] S. Alipour, A. T. Rezakhani, A. P. Babu, K. Mølmer, M. Möttönen, and T. AlaNissila. Correlation-Picture Approach to Open-Quantum-System Dynamics. arXiv:1903.03861 (to appear in PRX), 2020. URL https://arxiv.org/abs/1903.03861.

[54] K. Funo, N. Shiraishi, and K. Saito. Speed limit for open quantum systems. New J. Phys., 21:013006, 2019. DOI: 10.1088/13672630/aaf9f5.

[55] A. T. Rezakhani, D. F. Abasto, D. A. Lidar, and P. Zanardi. Intrinsic geometry of quantum adiabatic evolution and quantum phase transitions. Phys. Rev. A, 82:012321, 2010. DOI: 10.1103/PhysRevA.82.012321.

[56] S. Alipour and A. T. Rezakhani. Extended convexity of quantum Fisher information in quantum metrology. Phys. Rev. A, 91:042104, 2015. DOI: 10.1103/PhysRevA.91.042104.

[57] S. Alipour, F. Benatti, F. Bakhshinezhad, M. Afsary, S. Marcantoni, and A. T. Rezakhani. Correlations in quantum thermodynamics: Heat, work, and entropy production. Sci. Rep., 6:35568, 2016. DOI: 10.1103/PhysRevX.4.031042.

[58] R. W. Rendell and A. K. Rajagopal. Revivals and entanglement from initially entangled mixed states of a damped jaynescummings model. Phys. Rev. A, 67:062110, 2003. DOI: 10.1103/PhysRevA.67.062110.

[59] H. Carmichael. An Open Systems Approach to Quantum Optics. Springer, Berlin, 1993.

[60] J. G. Muga, X. Chen, S. Ibáñez, I. Lizuain, and A. Ruschhaupt. Transitionless quantum drivings for the harmonic oscillator. J. Phys. B: At. Mol. Opt. Phys., 43: 085509, 2010. DOI: 10.1088/0953-4075/43/8/085509.

[61] Christopher Jarzynski. Generating shortcuts to adiabaticity in quantum and classical dynamics. Phys. Rev. A, 88:040101, 2013. DOI: 10.1103/PhysRevA.88.040101.

[62] Adolfo del Campo. Shortcuts to adiabaticity by counterdiabatic driving. Phys. Rev. Lett., 111:100502, 2013. DOI: 10.1103/PhysRevLett.111.100502.

[63] S. Ibáñez, Xi Chen, E. Torrontegui, J. G. Muga, and A. Ruschhaupt. Multiple Schrödinger pictures and dynamics in shortcuts to adiabaticity. Phys. Rev. Lett., 109:100403, 2012. DOI: 10.1103/PhysRevLett.109.100403.

[64] Andrew Smith, Yao Lu, Shuoming An, Xiang Zhang, Jing-Ning Zhang, Zongping Gong, H. T. Quan, Christopher Jarzynski, and Kihwan Kim. Verification of the quantum nonequilibrium work relation in the presence of decoherence. New J. Phys., 20:013008, 2018. DOI: 10.1088/1367-2630/aa9cd6.

[65] L. Amico et al. Roadmap on atomtronics. 
arXiv:2008.04439, 2020. URL http://arxiv.org/ $\mathrm{abs} / 2008.04439$.

[66] A. del Campo and M. G. Boshier. Shortcuts to adiabaticity in a time-dependent box. Sci. Rep., 2:648, 2012. DOI: 10.1038/srep00648.

[67] Seth Lloyd. Universal quantum simulators. Science, 273:1073, 1996. DOI: 10.1126/science.273.5278.1073.

[68] Julio T. Barreiro, Markus Müller, Philipp Schindler, Daniel Nigg, Thomas Monz, Michael Chwalla, Markus Hennrich, Christian F. Roos, Peter Zoller, and Rainer Blatt. An open-system quantum simulator with trapped ions. Nature (London), 470:486, 2011. DOI: 10.1038/nature09801.

[69] Markus Müller, Sebastian Diehl, Guido Pupillo, and Peter Zoller. Engineered open systems and quantum simulations with atoms and ions. In Paul Berman, Ennio Arimondo, and Chun Lin, editors, Advances in Atomic, Molecular, and Optical Physics, Vol. 61 of Advances in Atomic, Molecular, and Optical Physics, pp. 1. Academic Press, 2012. DOI: 10.1016/B978-0-12396482-3.00001-6.

[70] I. M. Georgescu, S. Ashhab, and Franco Nori. Quantum simulation. Rev. Mod. Phys., 86:153, 2014. DOI: 10.1103/RevModPhys.86.153.

[71] R. Sweke, M. Sanz, I. Sinayskiy, F. Petruccione, and E. Solano. Digital quantum simulation of many-body non-Markovian dynamics. Phys. Rev. A, 94:022317, 2016. DOI: 10.1103/PhysRevA.94.022317. 